\title{
A Content-Based Search Engine on Medical Images for Telemedicine
}

\author{
David Cheung \& Chi-Hung Lee \\ Department of Computer Science \\ The University of Hong Kong \\ Hong Kong
}

\author{
Vincent $\mathrm{Ng}$ \\ Department of Computing \\ Hong Kong Polytechnic University \\ Hong Kong
}

\begin{abstract}
Retrieving images by content and forming visual queries are important functionality of an image database system. Using textual descriptions to specify queries on image content is another important component of content-based search. We describe a medical image database system MIQS which supports visual queries such as query by example and query by sketch. In addition, it supports textual queries on spatial relationships between the objects of an image. MIQS is designed as a client-server application in which the client access the database and its images via the $W W W$.
\end{abstract}

\section{Introduction}

Telemedicine [3, 4] has been rapidly developing in the last few years. Everyday, large number of valuable medical images are produced and physicians can retrieve similar images from databases to support their diagnosis and treatments. Therefore, medical image database systems which can be accessed through World Wide Web (WWW) are highly desirable. Moreover, a useful system must provide contentbased query for images and support queries on some non-content-based information associated with the images. In this paper, we present the design of MIQS (Medical Image Query System). It can be accessed via the WWW and it supports a) queries by example, b) queries by sketches, c) querying by spatial rules, so that users can specify conditions on spatial information which are difficult to do visually, d) keywords search on disease names and symptoms. The rest of this paper is organized as the follows: query specification model is presented in Section 2. A review of related works in content-based retrieval systems is presented in Section 3. The system architecture of MIQS is presented in Section 4. Section 5 is the conclusions.

\section{Query Specification Model}

In image database systems, there are generally two classes of the query [11]: 1) Query by external attributes and 2) Query by internal attributes.

\subsection{Query by external attributes}

Query by external attributes allows users to input the query according to the information associated with the images. For example, in medical image databases, the patient records are external attributes.

\subsection{Query by internal attributes}

Query by internal attributes allows users to specify queries according to the content of images. Generally, there are two types of content-based information:

1. Primitive information: It includes attributes such as color, texture and shape[1,2,6,7,12].

2. Logical information: It assumes that there are a number of objects in the images. The users can specify queries about properties of the objects and relationships between the objects $[5,8,14]$.

Many image database systems support querying by content by allowing users to form queries visually. Most of them support two types of visual query specifications: query by example and query by sketch. However, visual query specification has its shortcomings: 1. Difficult in specifying queries visually in some cases: "Find all images which satisfy the two conditions: (1) object $A$ is greater than object $B$; (2) for objects other than $A$ and $B$, their properties match those specified in the sketch".

2. Inflexible: Given an example image, there is no support for a user to adjust the properties of the objects in the example.

3. Ineffective: If the system does not have an example close to a user's target image, or the user has difficulty to input a good sketch, then it may not be effective to retrieve the target by either example or sketch.

Therefore, we suggest a new query specification method : query by spatial rules. In addition to visual specification, we provide a set of spatial rules for query specification. It allows the user to specify queries on: 1. Relative position between objects: Left of, Right of, Top of and Bottom of. The centroid of the objects are used for comparison. 
2. Spatial relationships between objects: Contain, Include, Overlap and Disjoint.

3. Insertion or removal of objects from the example images.

4. Comparison of the sizes of the objects and the distances between objects.

A set of reserved words are provided for users to specify this type of queries. This capability enhances immensely the query specification power.

\section{Related Works}

There are mainly two methods to specify contentbased queries in image databases: 1) use text to describe the content $[9,13] ; 2)$ use visual images to specify the queries. Some systems support image queries on primitive information like color and texture $[6,7,12]$. Some systems support the definition of objects in the images. VisualSEEk [5] supports both feature comparison and spatial query for unconstrained color images. In I2Cnet [10], user can input queries by examples and sketch. The selected example image or sketch is segmented into Regions of Interest (ROI) and specify queries based on the ROI's location, size, shape and texture. I2Cnet is one of the most comprehensive medical image database systems and it operates over the WWW. However, it has the following shortcomings: 1) It doesn't support queries on the relationships between the ROIs, 2) It doesn't support query by spatial rules and 3) It doesn't support query by external attributes which provides an important complementary searching mechanism. In the following, we will describe our approach adopted by MIQS in handling these issues. In particular, we will discuss how queries can be performed on content-based spatial rules.

\section{Architecture of MIQS}

MIQS uses the client-server architecture. MIQS clients interactive with the MIQS server as described in the architecture in Figure 1. In the server side, the database contains internal and external attributes of all the medical images, together with their URLs. When a user wants to find some images, he submits a query to a WWW browser by sketching the image or by finding out an example image which is similar to what he wants, also he can use the text input to supply supplementary information for the queries. Once the query arrives at the server, it processes the query to find the images which are the most similar to the query. The server then returns the locations (URLs) of the images to the user's WWW browser. Subsequently, the browser sends requests to the web sites to retrieve the images. Structurally, there are four components in MIQS : MIQS database, user-interface, the

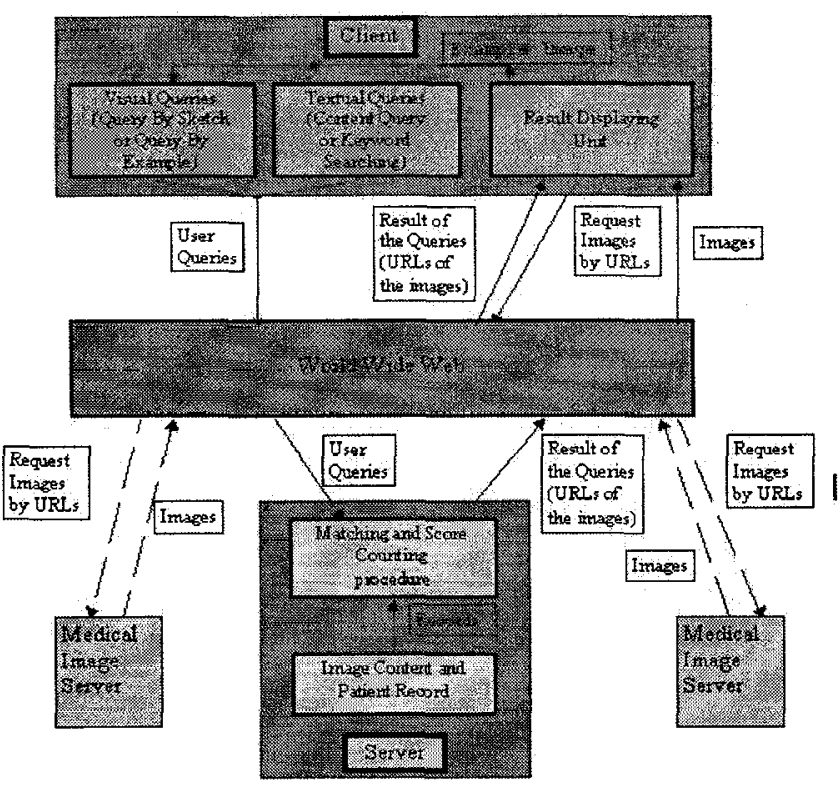

Figure 1:The architecture of the MIQS system.

matching and score counting, and the image display.

\subsection{MIQS Database}

The MIQS database stores the external and internal attributes of the images to support query processing. In addition, the URLs associated with the images are also stored in the MIQS database. In a medical image database system, in general, there are a number of objects in each image, and they can be classified as "known objects" and "unknown objects" [4]. The known objects are those that can be commonly found in all healthy human body, e.g., cerebrum, and cerebellum. Unknown objects are those that can be found only in some rare cases such as tumor and cancer.

\subsubsection{Database Creation}

In order to achieve good performance in the searching of similar images, we represent an object by its minimum bounding rectangle (MBR), and extract the attributes of the MBR to support searching. For example, the location, $x$-extent and y-extent of the MBR are important features of an object in a medical image. Other primitive features such as color and texture in general are not very useful in medical images because they mostly are very similar between same objects from different images. For every image, we extract and store the following attributes in the MIQS database : 1) The name, the starting value of $x$ and $y$, the $x$-extent and the $y$-extent of each object (its MBR) found in the image, 2) The disease and symptoms associated with the image and 3) The location (URL) of the image. 


\subsection{User Interface}

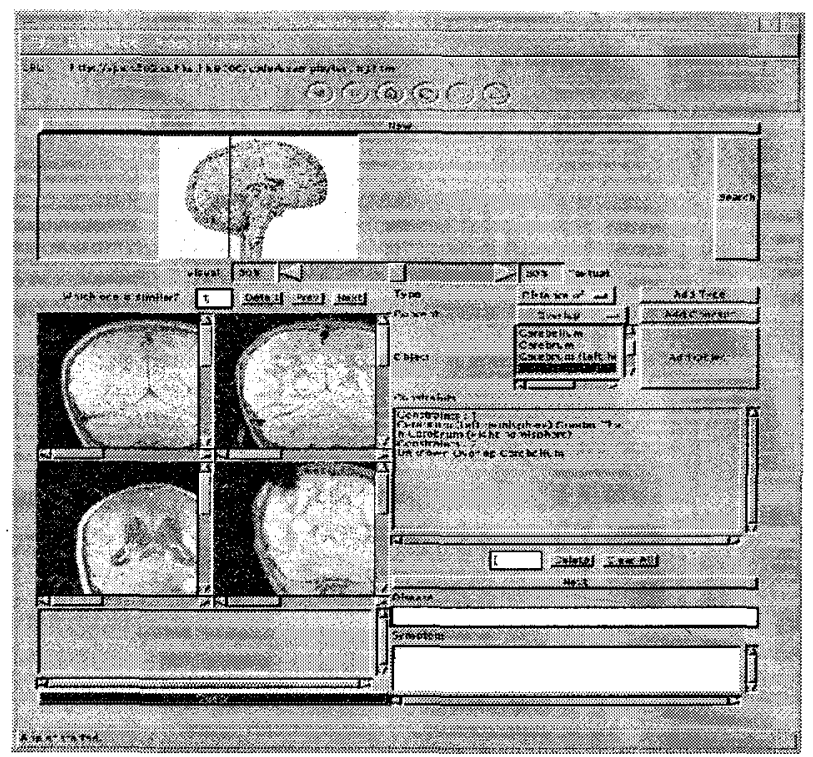

Figure 2: The query interface which supports query by example and query on spatial rules.

The MIQS client has two query interfaces. The first one support query by example and query by spatial rules. An example of the interface is presented in Figure 2. The second one supports query by sketch and query by spatial rules. Both interfaces have a visual query input window and a textual query input window. The upper region in the textual query input window supports query by spatial rules. Users can select spatial rules from a set of predefined rules to specify the spatial relationships between objects in an example or a sketch. We have defined more than twenty spatial rules in MIQS. The lower region supports query on external attributes on disease name and symptoms. Users input key words in this region to specify the query. Users can query the relationship between objects on size and distance. Moreover, they can specify query with spatial rules. The following is some examples on spatial rules :

1. Object $A$ is on the Left of Object B: the value of the $\mathrm{x}$ coordinate of the centroid of Object $\mathrm{A}$ is smaller than that of Object $\mathrm{B}$.

2. Object A contains Object B: the MBR of Object B is inside that of Object $A$.

3. Addition of Objects: Inserts objects into the query example or sketch.

The visual query regions are very different in these two interfaces.

1. Query by example: The top window displays the image of a body part under study. An user can use specify a cross section he is interested in. A set of example images will be shown on the displaying windows. The user can then choose an image which is close to his target query for further query.

2. Query by sketch: An user can use two different ways to produce a sketch. He can ise a tool to draw a sketch, or he can retrieve an image from the web as a sketch.

Either way, after the visual component is specified, together with the textual component, the query will be submitted to the MIQS server. The images returned are shown on the displaying windows. In addition, the user can adjust the relative significance of the visual queries verse that of the textual queries. If a user thinks that the example image or the sketch is very close to his target, then he can increase the weighting on the visual queries and vice versa.

\subsection{Matching and Score Counting}

We will explain the matching mechanism in MIQS which compares the attributes of a target image (IM) in the database against a query specification (SI) and returns a score on the similarity of the target image. The similarity consists of two parts. As has been discussed, SI has a visual query part (example or sketch image), and a textual query part (spatial rules, disease and symptom specification). A similarity function $d_{1}=D_{v}(S I, I M)$ will be used to measure the difference between the objects in the visual parts of SI and the objects in IM. Another similarity function $d_{2}=D_{t}(S I, I M)$ will be used to compare the attributes of IM against the conditions set in the textual query part of SI. The score $d_{2}$ will measure the number of conditions in the textual query that can be satisfied by the attributes of IM. At the end, the similarity of IM against SI is given by

$$
\operatorname{sim}(S I, I M)=w_{1} * d_{1}+w_{2} * d_{2},
$$

where $w_{1}$ and $w_{2}$ are two adjustable weightings. The matching component will return those images that have the best similarity (small values) with respect to the query specification.

\subsubsection{Visual query part}

Suppose there are $\mathrm{n} 1$ known objects $o_{1}, \ldots, o_{n 1}$, and $\mathrm{n} 2$ unknown objects $u_{1}, \ldots, u_{n 2}$ in the query specification SI, and the target image IM has $\mathrm{m} 1$ known objects $O_{1}, \ldots, O_{m 1}$, and $\mathrm{m} 2$ unknown objects $U_{1}, \ldots, U_{m 2}$.

We will first explain how to define the measure $d_{1}=D_{v}(S I, I M)$. For any object in an image, MIQS considers four features : $\mathrm{x}$-start and $\mathrm{y}$-start of the object (MBR), and its $\mathrm{x}$ - and y-extent. A distance $d\left(o_{i}, O_{i}\right)$ between an object $o_{i}$ in SI and an object $O_{i}$ in IM is a sum of the differences of their four features. The difference of a feature such as the $\mathrm{x}$-start is their city-block distance. The difference of x-extent 
or y-extend is their absolute difference. In the simplest case, SI and IM have the same number of known and unknown objects, i.e., $n_{1}=m_{1}$ and $n_{2}=m_{2}$, the known objects in IM are matched 1-to-1 to those in SI by their names, and the unknown objects also have a 1-to-1 mapping. Therefore we can measure the distance between IM and SI by defining

$d_{1}=D_{v}(S I, I M)=\sum_{i=1}^{n 1} d\left(o_{i}, O_{i}\right)+\sum_{i=1}^{n 2} d\left(u_{i}, U_{i}\right)$.

If a known object $o_{i}$ in SI has no corresponding object in IM, then MIQS assumes there is a "dummy" object $O_{i}$ in IM corresponding to $o_{i}$. The distance $d\left(o_{i}, O_{i}\right)$ will be a system defined adjustable constant which should be relative large so that target image with no corresponding object will be penalized. As for the unknown objects, even if SI and IM have the same number of objects, there could be many different mappings between them. Since in practice, there should not be many unknown objects, MIQS exhaustively tries out all possible mappings and select the one with the minimum distance. If SI has more unknown objects, then MIQS will again assign dummy objects in IM to match with them.

\subsubsection{Textual query part}

Both internal and external attributes of the target image IM are checked with the conditions in processing the textual query. For the spatial rules, the internal attributes must satisfy the rules precisely. For the query on the external attributes, some allows partial matching. For example, a score could be computed on the ratio of matching symptoms specified in the query. As discussed before, the similarity function $d_{2}=D_{t}(S I, I M)$ will return a score on the number of rules and external attributes satisfied by IM.

\section{Conclusion}

In this paper, we have presented the design and architecture of MIQS, a Web-based medical image database system, which allows users to perform content-based queries such as query by example and sketch. In addition, it supports a novel query by content method of using spatial rules. MIQS is designed to support telemedicine, its client is implemented in Java which can be run on any platform. The user can use a browser to submit queries to the MIQS server and the required images will be returned via the Web.

\section{References}

[1] V. Ng, D.-W. Cheung and A. Fu, Medical Image Retrieval by Color Content, Proc. 1995 IEEE Intl. Conf. On System, Man and Cybernetics, Vancouver, Canada. (Oct. 1995)

[2] V. Ng, D.W. Cheung and A. Fu, Adaptive Cólor Histogram Indexing, Proc. SPIE Intl. Sympo- sium on Voice, Video, and Data Communication, SPIE Proc. V.2606-20, Philadelphia, Pennsylvania, U.S.A. (Oct. 1995)

[3] F. Williams and M. Moore, Telemedicine: Its place on the information highway, http:// naftalab.bus.utexas.edu/nafta-7/telepap.html.

[4] M. Moore, Elements of Success in Telemedicine Projects, http://naftalab.bus.utexas.edu/nafta7/elemsucc.html.

[5] J. R. Smith and S.-F. Chang, VisualSEEk: a fully automated content-based image query system, ACM Multimedia '96, November, 1996.

[6] W. Niblack, R. Barber, W. Equitz, M. Flickner, E. Glasman, D. Petkovic, P. Yanker, and C. Faloutsos, The QBIC project: Querying images by content using color, texture, and shape, In Storage and Retrieval for Image and Video Databases; volume SPIE Vol. 1908, February 1993.

[7] Virage, Inc., VIR TECHNOLOGY DEMO, http:// www.virage.com/cgi-bin/random.

[8] E. G. M. Petrakis and C. Faloutsos, Similarity searching in large image databases, Technical Report 3388, Dept. of Comp. Sci., Uni. of Maryland, 1995.

[9] V. E. Ogle and M. Stonebraker, Chabot: Retrieval from a Relational Database of Images, IEEE Computer, pages 40- 48, September, 1995.

[10] S. C. Orphanoudakis, C. E. Chronaki, and D. Vamvaka, I2Cnet:Content-based Similarity Search in Geographically Distributed Repositories of Medical Images, http://www.ics. forth.gr/ telemed/papers/i2cnet/paper1.html.

[11] V. N. gudivada and V. V. Raghavan, Contentbased Image Retrieval Systems, IEEE Computer, pages 18-22, September, 1995.

[12] Informix Software Co., Visual Intelligence Demo., http://www.informix.com/virdemo/cgibin/Webdriver?MIval=demos

[13] R. K. Srihari, Automatic Indexing and ContentBased Retrieval of Captioned Images, IEEE Computer, page 49-56, September, 1995.

[14]. P. W. Huang and Y. R. Jean, Design of Large Intelligent Image Database Systems, Intl. Journal of Intelligent Systems, Vol. 11, 347-365 (1996). 